\title{
MicroRNA-181a-mediated downregulation of AC9 protein decreases intracellular cAMP level and inhibits ATRA-induced APL cell differentiation
}

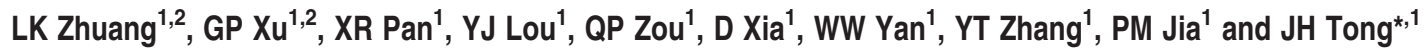

AC9 is one of the adenylate cyclase (AC) isoforms, which catalyze the conversion of ATP to CAMP, an important second messenger. We previously found that the integration of CAMP/PKA pathway with nuclear receptor-mediated signaling was required during all-trans retinoic acid (ATRA)-induced maturation of acute promyelocytic leukemia (APL) cells. Here we showed that AC9 could affect intracellular CAMP level and enhance the trans-activity of retinoic acid receptor. Knockdown of AC9 in APL cell line NB4 could obviously inhibit ATRA-induced differentiation. We also demonstrated that miR-181a could decrease AC9 expression by targeting $3^{\prime}$ UTR of AC9 mRNA, finally controlling the production of intracellular cAMP. The expression of miR-181a itself could be inhibited by CEBP $\alpha$, probably accounting for the differential expression of miR-181a in NB4 and ATRA-resistant NB4-R1 cells. Moreover, we found that AC9 expression was relatively lower in newly diagnosed or relapsed APL patients than in both complete remission and non-leukemia cases, closely correlating with the leukemogenesis of APL. Taken together, our studies revealed for the first time the importance of miR-181a-mediated AC9 downregulation in APL. We also suggested the potential value of $\mathrm{AC} 9$ as a biomarker in the clinical diagnosis and treatment of leukemia.

Cell Death and Disease (2014) 5, e1161; doi:10.1038/cddis.2014.130; published online 10 April 2014

Subject Category: Cancer

The second messenger cAMP has been demonstrated to have an important role in cell proliferation, differentiation and apoptosis. The formation of intracellular cAMP from ATP is catalyzed by a family of enzymes, adenylate cyclases (ACs). Currently, nine membrane-associated members of mammalian $A C(A C 1-9)$ have been identified. Most of them are widely expressed, while AC1 and AC3 are mainly found in the brain. ${ }^{1}$ Such a broad distribution indicates that a given cell may contain multiple AC isoforms. The alterations in the expression of $A C$ isoforms usually affect the intracellular cAMP concentration that controls a cascade of transcriptional events and regulates the fate of cells. As the mechanisms underlying the expression regulation and the activity of $A C$ are distinct between different isoforms, it is important to define the unique regulatory properties of each AC family member.

Along with other groups, we previously reported that the activation of cAMP/PKA-dependent pathway was an indispensable event in the molecular mechanism of all-trans retinoic acid (ATRA)-induced differentiation therapy for acute promyelocytic leukemia (APL), ${ }^{2-5}$ a class of human myeloid leukemia characterized by the differentiation block of granulopoiesis at the promyelocytic stage. This point of view was based on several evidences. First, it was found that the basal level of intracellular CAMP was relatively higher in the retinoic acid (RA)-sensitive APL cell line NB4 than in the RA-resistant subclone NB4-R1. ${ }^{3}$ Second, the cAMP-elevating agents could not only cooperate with RA to trigger the terminal differentiation of NB4-R1 cells and overcome their resistance to RA, but also render the NB4 cells even responsive to physiological levels of RA. ${ }^{4}$ On the contrary, disrupting the cAMP signaling with some cAMP antagonists could result in a reversible resistance of NB4 cells to pharmacological levels of RA. $^{5}$ Therefore, it was concluded that the ATRA-induced differentiation process in APL cells was associated with the intracellular CAMP level. Further studies suggested that the discrepancy of AC activation between NB4 and NB4-R1 may contribute to the different cAMP levels in these two cell lines, resulting in their different sensitivity to ATRA-induced maturation. ${ }^{3}$ Nevertheless, the molecular basis involved in the control of expression and activation of AC in NB4 and NB4-R1 cells remained unknown.

Recently, with a survey of gene expression profile by using whole-genome cDNA chip analysis, we found a relatively high level of AC9 isoform in NB4 cells. Human AC9 is a particular

\footnotetext{
${ }^{1}$ State Key Laboratory of Medical Genomics, Faculty of Medical Laboratory Science and Shanghai Institute of Hematology, Rui-jin Hospital, Shanghai Jiao Tong University School of Medicine, Shanghai, China

${ }^{*}$ Corresponding author: JH Tong, State Key Laboratory of Medical Genomics, Faculty of Medical Laboratory Science and Shanghai Institute of Hematology, Rui-jin Hospital, Shanghai Jiao Tong University School of Medicine, 197 Rui-jin Er Road, Shanghai 200025, China. Tel/Fax: +86 21 64454908; E-mail: tongjh@ hotmail.com

${ }^{2}$ These authors contributed equally to this work.

Keywords: AC9; miR-181a; CAMP; APL; cell differentiation

Abbreviations: AC, adenylate cyclase; ATRA, all-trans retinoic acid; APL, acute promyelocytic leukemia; RA, retinoic acid; miRNA, microRNA; CLL, chronic lymphocytic leukemia; ChIP, chromatin immunoprecipitation; RAR, retinoic acid receptor; NBT, nitroblue-tetrazolium; CR, complete remission; 1,25D, 1,25-dihydroxyvitamin D3; wt, wild type; mut, mutant

Received 29.11.13; revised 27.2.14; accepted 28.2.14; Edited by E Candi
} 
member of the AC family, because of its divergent sequence with other members and its insensitivity to forskolin. ${ }^{6,7}$ It was indicated that AC9 was expressed in various organs, especially in skeletal muscle, brain, lung and heart., Although great progress has been made in our understanding of the complexity of AC signaling, AC9 is the least characterized isoform of the family. In addition to its activation by stimulatory $G$ protein and its inhibition by $\mathrm{Ca} 2+$ /calcineurin, ${ }^{6,7}$ little is known about the regulatory properties of AC9. Most recently, Huang et al. ${ }^{8}$ reported that miR-142-3p could restrict cAMP production in certain T cells by targeting AC9 mRNA, suggesting that the microRNA (miRNA) may control AC expression and the final level of intracellular CAMP in various types of cells.

MiRNAs are naturally occurring, non-coding RNA molecules of 20-25 nucleotides that mediate the expression regulation of target genes at post-transcriptional level by inducing translational repression or mRNA degradation. ${ }^{9}$ Over the past years, the research showing the involvement of miRNAs as a kind of epigenetic regulation in a variety of cell processes has been markedly increasing. Deregulation of miRNA expression has been considered to be a key cause in the pathogenesis of human diseases including cancer. In hematopoietic malignancies, a number of miRNAs have been reported to have a tumor-suppressive or oncogenic role in leukemogenesis. ${ }^{10-13}$ For instance, miR-15/miR-16-1 was found to be frequently downregulated in chronic lymphocytic leukemia (CLL), which is characterized by malignant $B$ cells overexpressing the anti-apoptotic BCL-2 protein. ${ }^{14,15}$ The negative regulation of $B C L-2$ by $\mathrm{miR}-15 / \mathrm{miR}-16-1$ in $\mathrm{B}-\mathrm{CLL}$ represented the first connection between disturbed miRNA expression and leukemia. ${ }^{12,16}$

In this study, we intensively explore the functions of AC9 in ATRA-induced APL cell differentiation and provide evidences supporting the critical roles of CAMP/PKA pathway in the therapeutic action of ATRA on APL. Meanwhile, we find a miRNA-based regulatory mechanism underlying the AC9 expression and first suggest the potential value of AC9 in the clinical diagnosis and treatment of leukemia.

\section{Results}

AC9 protein affects intracellular cAMP level and enhances the trans-activity of RA receptor on target genes. Our previous cDNA chip assay (data not shown) and RT-PCR analysis (Supplementary Figure S1) showed that two isoforms, AC9 and AC7, were expressed in NB4 cells. Here, we first examined by western blot the expression of these two isoforms in NB4 and NB4-R1 cells, respectively. We found that the level of AC9 protein was significantly lower in NB4-R1 cells than in NB4 cells (Figure 1a). Unfortunately, we failed to detect the AC7 protein by using the commercially available antibodies. Therefore, we tried to focus our study on AC9 isoform as well as its potential roles in APL cell differentiation. The AC9 expression plasmid and two si-AC9 plasmids were then respectively transfected into 293T cells. By using ELISA assay kit, we measured that the intracellular cAMP content was obviously increased in AC9-transfected cells when compared with that in control cells (Figure 1b). In addition, this increasing effect of AC9 protein could be suppressed by both psi-AC9-(1) and psi-AC9-(2) (Figure 1c), strongly indicating that AC9 protein could directly affect intracellular cAMP level.

As it has been recognized that CAMP/PKA pathway was integrated into ATRA-induced differentiation process in APL cells probably through promoting the transcriptional activation of RA receptor (RAR) $\alpha$-regulated genes, ${ }^{3}$ we next examined the effect of AC9 protein on trans-regulatory activity of RAR $\alpha$. Transient transfection with DR5 RARE-containing reporter gene showed that ATRA-induced trans-activity of RAR $\alpha$ could be enhanced about twofold when AC9 protein was coexpressed in the cells (Figure 1d), whereas either of the two si-AC9 could obviously impair such enhancing effect of AC9 on transactivation of $\operatorname{RAR} \alpha$ (Figure 1e). These data further supported our idea that AC9 was involved in CAMP/PKA pathway cooperating with $\mathrm{RAR} \alpha$-mediated signaling during ATRA-induced APL cell maturation.

AC9 knockdown attenuates ATRA-induced NB4 cell differentiation. To investigate the potential roles of AC9 protein in ATRA-induced APL cell differentiation, we generated two stable AC9-knockdown cell lines, NB4-siAC9-1 and NB4-siAC9-2, by using the lentiviral vector-based RNA interference approach. Western blot showed the AC9 protein in varying degrees of decline in these two cell lines when compared with the control cells NB4-siNC (Figure 2a). The effects of AC9 knockdown on ATRA-induced cell differentiation were thus evaluated according to CD11b expression, nitroblue-tetrazolium (NBT) reduction ability and morphological observation, respectively. As a result, although ATRA-induced cell maturation seemed to proceed faster in siRNA-transfected NB4 cells than in parent NB4 cells, we found a significant decrease of CD11b expression and NBT reduction ability in AC9-knockdown NB4 cells compared with that in the control cells NB4-siNC (Figures $2 b$ and $c$ ). In addition, under the same treatment with ATRA, morphological features of typical maturation could be clearly observed in NB4-siNC cells, but not evidently in NB4-siAC9 cells (Figure 2d), fully indicating the importance of AC9 protein in ATRA-induced NB4 cell differentiation.

miR-181a targets $3^{\prime}$ UTR of AC9 mRNA and decreases AC9 protein level. The question was then raised: what was it that caused the differential expression of AC9 in NB4 and NB4-R1 cells? To answer this question, we conducted an exploration on the molecular mechanisms underlying the expression regulation of AC9. During the past decade, miRNA has been found to be one of the most important post-transcriptional regulators fine-tuning gene expression in a sequence-specific manner. By means of three different algorithms, including PicTar, Targetscan and microRNA.org, we predicted several candidate miRNAs probably pairing with the complementary sites in the $3^{\prime}$ UTR of AC9 mRNA (Figure $3 a$ ). Meanwhile, microarray analysis showed that, among these candidates, miR-181a exhibited about twofold higher level in NB4-R1 cells than in NB4 cells (Figure 3b), having an inverse relationship with the AC9 protein level in these two cell lines. This significant differential expression of miR-181a in NB4 and NB4-R1 cells was then verified by both ordinary and real-time quantitative RT-PCR (Figures $3 c$ and d), 
a

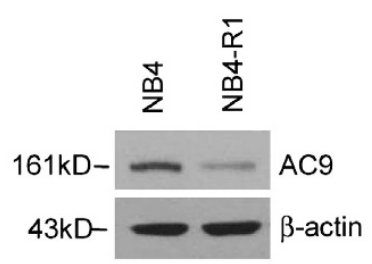

C

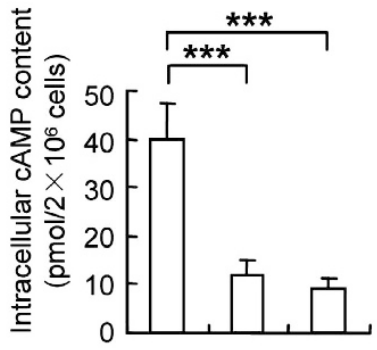

b
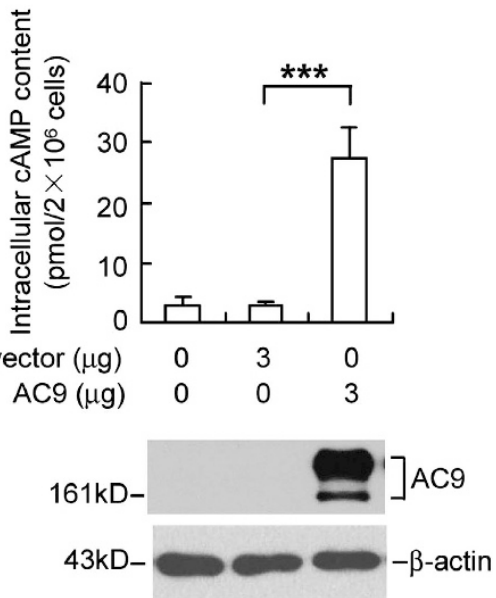

d $\square$-ATRA $(1 \mu \mathrm{M})$
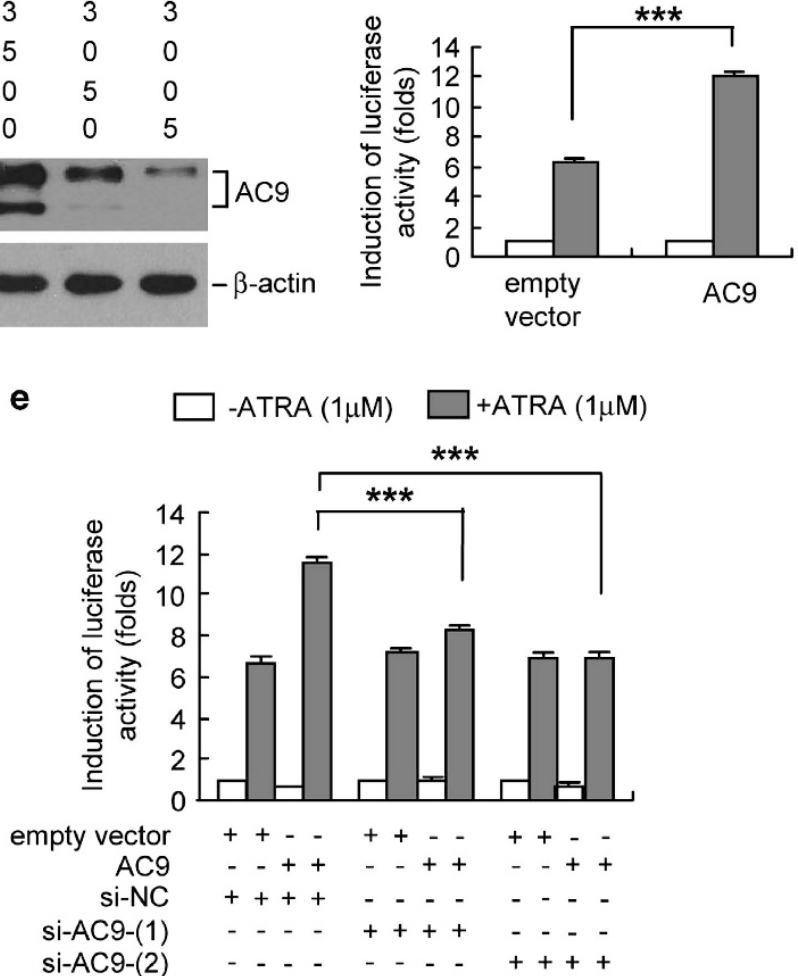

Figure 1 Effects of AC9 protein on intracellular cAMP level and transactivation of RARE-containing reporter gene. (a) Western blot analysis of AC9 expression in NB4 and NB4-R1 cells. $\beta$-Actin expression was used as a loading control. (b) Determination on intracellular CAMP concentration and AC9 protein level in AC9-transfected 293T cells. $\beta$-Actin expression was used as a loading control. Each value represents the mean \pm S.D. of three independent experiments. ${ }^{* \star \star} P<0.001$. (c) Two si-AC9 plasmids were respectively co-transfected with AC9 expression plasmid into 293T cells. Intracellular CAMP content and AC9 protein level were detected in these cells. psi-NC was used as a negative control. $\beta$-Actin expression was used as a loading control. Each value represents the mean \pm S.D. of three independent experiments. ${ }^{* * *} P<0.001$. (d and $\mathbf{e}$ ) RARE-TK-luciferase reporter plasmid was co-transfected with the indicated plasmids into COS7 cells. At $12 \mathrm{~h}$ after transfection, cells were treated with or without $1 \mu \mathrm{mol} / /$ ATRA. Luciferase activity was measured $36 \mathrm{~h}$ after the transfection. Each value represents the mean \pm S.D. of three independent experiments. ${ }^{* \star \star} P<0.001$

further implying the potential role of miR-181a in the regulation of AC9 expression.

We then performed a detailed analysis by using a reporter vector system to explore whether miR-181a targeted $3^{\prime}$ UTR of AC9 mRNA. Two luciferase reporter constructs containing predicted miR-181a-binding sequences (wild type (wt) and mutant (mut), respectively) in AC9 $3^{\prime}$ UTR were prepared (Figure 4a). After transient transfection into 293T cells, we found that miR-181a could significantly inhibit the expression of the reporter gene containing wt-AC9 $3^{\prime}$ UTR, but not mut-AC9 $3^{\prime}$ UTR nor the empty control (Figure 4b). Moreover, we noted that transfection with miR-181a could decrease the 
a

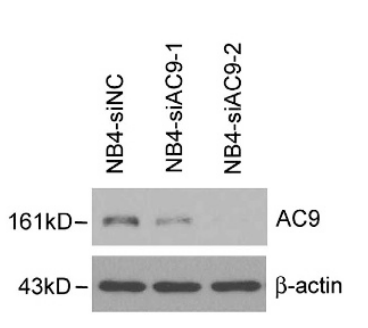

c

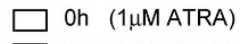

$$
\begin{aligned}
& 24 \mathrm{~h}(1 \mu \mathrm{M} \text { ATRA }) \\
& \text { 36h }(1 \mu \mathrm{M} \text { ATRA) }
\end{aligned}
$$
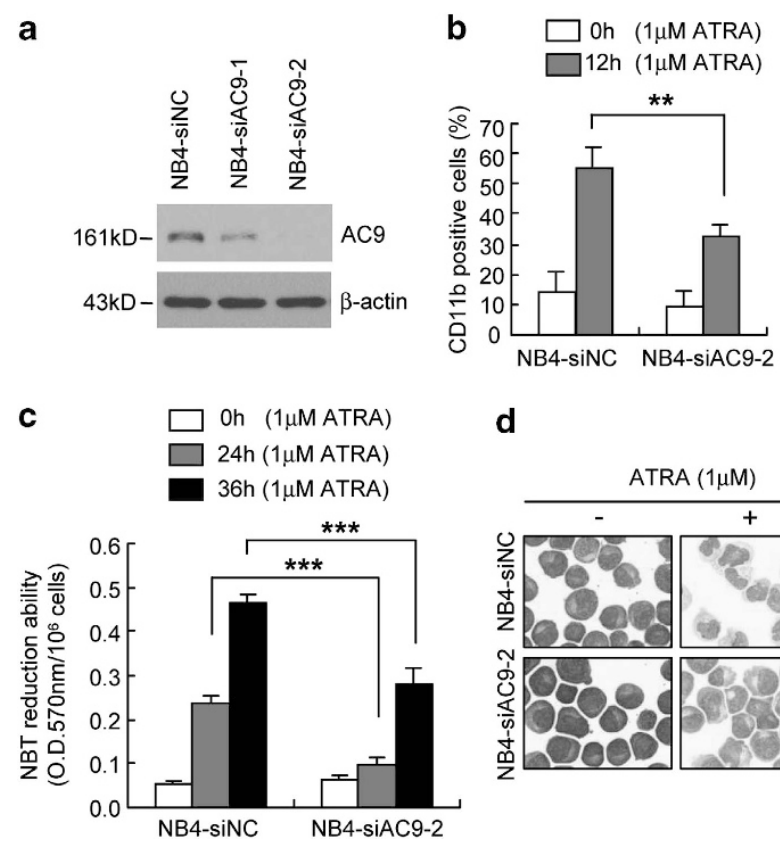

d

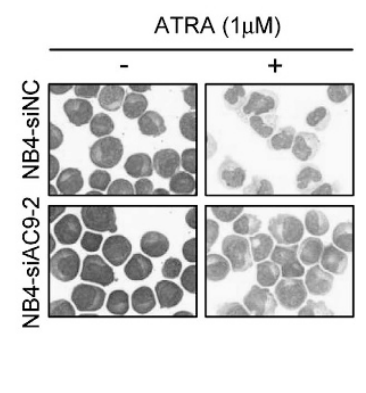

Figure 2 Roles of AC9 in ATRA-induced NB4 cell differentiation. (a) siRNAs were stablely transfected into NB4 cell lines as indicated. Their inhibitory effects on AC9 expression levels were analyzed by western blot. $\beta$-Actin expression was used as a loading control. ( $b$ and $\mathbf{c}$ ) CD11b expression and NBT reduction ability of NB4siNC and NB4-siAC9-2 cells following the indicated treatments were examined. Each value represents the mean \pm S.D. of three independent experiments. ${ }^{* \star} P<0.01$; ${ }^{* \star} P<0.001$. (d) Morphological features of NB4-siNC and NB4-siAC9-2 cells were shown after exposure to $1 \mu \mathrm{mol} / \mathrm{l}$ ATRA for $36 \mathrm{~h}$

levels of both exogenous and endogenous AC9 protein, as well as the intracellular cAMP production (Figures $4 \mathrm{c}-\mathrm{e}$ ). In consistent with this, miR-181a could also abolish the AC9-enhanced RAR $\alpha$ trans-regulatory activity (Figure 4f), similar with the effects of si-AC9 (Figure 1e). These results strongly confirmed that AC9 was a target for miR-181a, and indicated that the negative regulation of miR-181a on AC9 protein expression should be closely related with the intracellular cAMP level.

miR-181a expression can be repressed by CEBP $\alpha$. In an attempt to further uncover the transcriptional regulation of miR-181a, we analyzed the sequences in miR-181a gene promoter by using TransFac software (http://www.generegulation.com/pub/programs.html). Two putative binding sites of CEBP $\alpha$ were revealed upstream of the pre-miR-181a sequences. We then constructed a series of reporter plasmids containing different fragments within the miR-181a promoter (Figure 5a) to determine the effect of CEBP $\alpha$ on miR-181a expression regulation. Consequently, the reporter construct without $\mathrm{CEBP} \alpha$-binding sites had no response to $\operatorname{CEBP} \alpha$, similar to the empty pXP2 vector. By contrast, the reporter genes, containing whether one or two CEBP $\alpha$-binding sites, all showed about $80-90 \%$ of transcriptional repression when $\mathrm{CEBP} \alpha$ was co-transfected into the cells (Figure 5b). These results were then strengthened by quantitative RT-PCR data that enforced CEBP $\alpha$ could markedly decrease the miR-181a expression in 293T cells (Figure 5c). Chromatin immunoprecipitation (ChIP) assay further confirmed that both of

these two CEBP $\alpha$-binding sites in the miR-181a promoter presented their effective binding abilities to CEBP $\alpha$ protein (Figure 5d). Moreover, the western blot showed that the expression of CEBP $\alpha$ in NB4 cells was indeed higher than that in NB4-R1 cells (Figure 5e). All these results fully indicated that CEBP $\alpha$ could negatively regulate miR-181a expression, probably accounting for the differential levels of miR-181a in NB4 and NB4-R1 cells.

Expression levels of miR-181a and AC9 are associated with the leukemogenesis of APL patients. In this study, we also detected the expression of miR-181a and AC9 in primary bone marrow cells from APL patients. A total of 10 APL patients, including 3 newly diagnosed (patient \#1, \#9 and \#14), 3 with complete remission (CR) (patient \#15, \#19 and \#22) and 4 relapsed (patient \#8, \#13, \#24 and \#25), were first collected and analyzed by RT-PCR for miR-181a and AC9 expression, respectively. The results showed that miR-181a expression was relatively higher in newly diagnosed or relapsed patients than in CR patients, whereas AC9 expression was just the opposite (Figures 6a and b). On this basis, we increased the number of patient samples. In all, 62 APL patients (21 newly diagnosed, 19 with $\mathrm{CR}$ and 22 relapsed) and 15 non-leukemia patients were then evaluated for miR-181a expression by real-time quantitative PCR. As expected, a relatively higher expression of miR-181a was found in newly diagnosed or relapsed patients, but a very low expression in both non-leukemia patients and APL patients with CR (Figure 6c). A significant inverse correlation between miR-181a and AC9 expression was observed (Figure 6d). However, no inverse relationship was found between miR-181a and CEBP $\alpha$ expression, although the latter was higher in newly diagnosed patients than in other three groups (Supplementary Figure S2). Our results indicated that both miR-181a and AC9 expression levels were correlated well with the leukemogenesis of APL patients, implying that the level of miR-181a or AC9 in primary bone marrow cells may function as biomarkers for diagnosis and prognosis of APL.

\section{Discussion}

Since the characterization of a gene family encoding AC isoforms, great advances have been made in our understanding of the control of intracellular CAMP concentrations, which usually accompany different drug responses and influence the treatment effects of diseases. Here, we provided experimental evidences that AC9 isoform had an important role in APL cell differentiation. Knockdown of AC9 could significantly decrease intracellular CAMP level and inhibit ATRA-induced NB4 cell differentiation. This finding was also sustained by the results that the AC9 expression was relatively higher in APL patients with CR or non-leukemia patients than in newly diagnosed or relapsed ones, suggesting that AC9 expression was required for the action of ATRA and the deficiency of AC9 may be associated with leukemogenesis.

However, it should be pointed out that, inconsistent with what we observed in NB4 and NB4-R1 cells, we did not find any significant differences in AC9 expression level between the newly diagnosed APL patients and the relapsed ones who frequently resisted the ATRA treatment. Several points may 


\begin{tabular}{|c|c|}
\hline $\begin{array}{c}\text { microRNAs predicted } \\
\text { to target AC9 }\end{array}$ & Pictar Score \\
\hline hsa-miR-7 & 4.91 \\
\hline hsa-miR-181a & 2.52 \\
\hline hsa-miR-181b & 2.52 \\
\hline hsa-miR-181c & 2.52 \\
\hline hsa-miR-142-3p & 1.55 \\
\hline
\end{tabular}

c

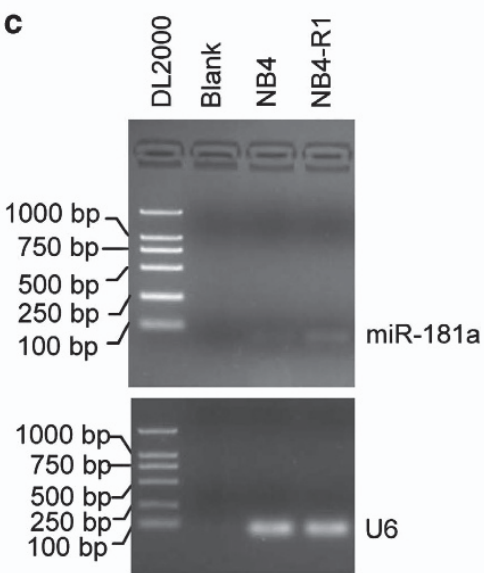

b

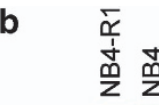

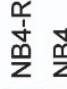

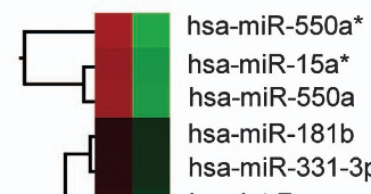

hsa-let-7a

hsa-miR-185

hsa-miR-2861

hsa-miR-196b

hsa-miR-494

hsa-miR-26a

hsa-miR-30d

hsa-miR-374b

hsa-miR-638

hsa-miR-181a

hsa-miR-196b

hsa-let-7b

hsa-miR-22

d

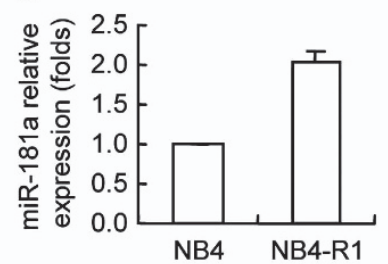

-3.00
-2.00
-1.00
0.00
1.00
2.00
3.00

Figure 3 Microarray and PCR analysis of miR-181a expression in NB4 and NB4-R1 cells. (a) Partial prediction results of miRNAs targeting AC9 by three algorithms. (b) Partial miRNA profiles of NB4 and NB4-R1 cells by microarray. Red color indicates high expression and green color indicates low expression, according to the color bar shown on the right in logarithmic scale. (c and d) Analysis of miR-181a expression in NB4 and NB4-R1 cells by ordinary (c) and real-time quantitative RT-PCR (d). Each value represents the mean \pm S.D. of three independent experiments

be taken into account. First, the RA-resistant NB4-R1 cell line, derived from the same APL patient as the RA-sensitive NB4 cell line, offers a cellular model to elucidate the molecular mechanisms underlying RA resistance, ${ }^{17}$ but this particular case may not reflect the whole RA-resistant group of APL patients. Second, besides the different basal level of CAMP in NB4 and NB4-R1 cells, we previously reported a rapid induction of $\mathrm{CAMP}$ level within a few minutes after treatment with ATRA in NB4 cells and fresh APL cells, but not in NB4-R1 cells. ${ }^{3}$ Nevertheless, no obvious upregulation of AC9 expression was detected in both NB4 and NB4-R1 cells after ATRA treatment (data not shown). Thus, the resistance of NB4-R1 cells to ATRA-induced maturation should not be simply attributed to the deficiency in AC9 expression. Whether there were some defects in the initiation of AC9 activation in NB4-R1 cells remained to be further explored.

In this study, we also showed the evidences for AC9 regulation by miR-181a. miR-181a was one of the first miRNAs demonstrated to be highly expressed in hematopoietic tissues. $^{12}$ In mice, miR-181a was detected in early progenitor cells and dynamically upregulated during B-cell commitment. The ectopic expression of miR-181a could lead to a proliferation in B-lymphoid lineage. ${ }^{18}$ Afterward, increasing evidences have indicated that miR-181 family could function as both positive and negative regulators during cell differentiation. For instance, it was reported that miR-181a was decreased in 1,25-dihydroxy-vitamin D3 (1,25D)-induced differentiation of HL60 and U937 cells, and transfection of miR-181a in 1,25D-treated cells could reduce G1 block and abrogate the expression of monocytic differentiation markers. ${ }^{19}$ Similar downregulation of $\mathrm{miR}-181 \mathrm{~b}$ was also found in NB4 cells during ATRA treatment, ${ }^{20}$ suggesting that miR-181 may contribute to the malignant phenotype in myeloid lineage. However, in another retrospective study conducted on cytogenetically normal AML patients, Marcucci et al. $^{21}$ showed an inverse correlation between miR-181 expression and poor clinical outcome. In addition to the research on leukemogenesis, the opposing roles of miR-181 were also described in solid tumor development, progression and drug response of cancer cells. ${ }^{22-25}$ Now, it is considered that the complexity of miRNA roles in cellular processes is 
a

\begin{tabular}{|c|c|c|c|}
\hline pCMV4 & luciferase & polyA & control \\
\hline pCMV4 & luciferase & wt-AC9 3'UTR polyA & wt \\
\hline pCMV4 & luciferase & mut-AC9 3'UTR polyA & mut \\
\hline
\end{tabular}

b $\square$ empty vector $\square$ miR-181a

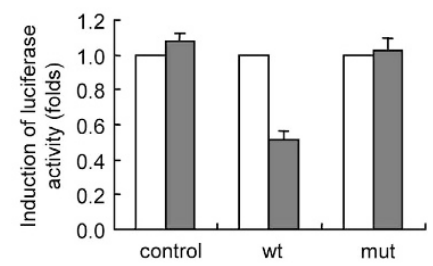

c

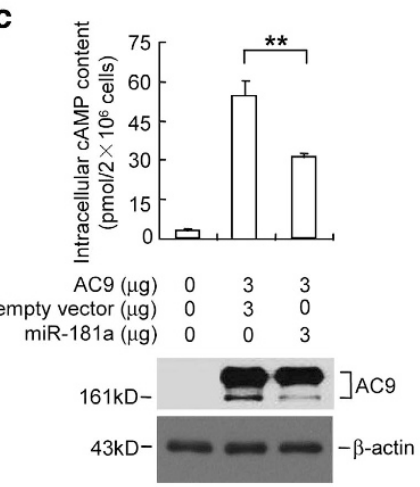

d

empty vector $(\mu \mathrm{g}) 1 \quad 0 \quad 5 \quad 0 \quad 10 \quad 0$ $\operatorname{miR}-181 \mathrm{a}(\mu \mathrm{g}) 0 \quad 1 \quad 0 \quad 5 \quad 0 \quad 10$

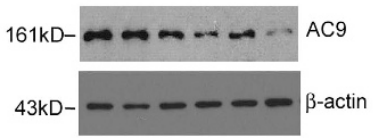

e

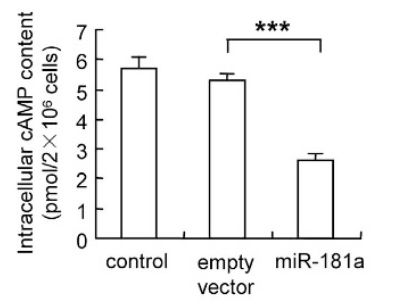

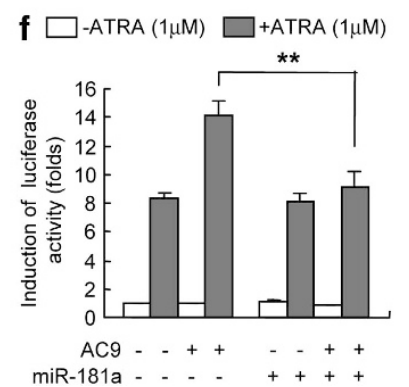

Figure 4 Effects of miR-181a on AC9 expression and intracellular cAMP content. (a) Schematic representation of wt and mut-AC9 $3^{\prime}$ UTR-containing reporter constructs. (b) Effects of miR-181a on the expression of AC9 $3^{\prime}$ UTR-containing reporter genes. Luciferase activity was shown relative to the values obtained in the cells co-transfected with empty vector and the same reporter construct. Each value represents the mean \pm S.D. of three independent experiments. (c) Intracellular cAMP and AC9 expression levels were measured in 293T cells transfected with the indicated plasmids. $\beta$-Actin expression was used as a loading control. Each value represents the mean \pm S.D. of three independent experiments. ${ }^{* \star} P<0.01$. (d) Western blot analysis of endogenous AC9 expression in HT1080 cells transfected with the indicated plasmids. $\beta$-Actin expression was used as a loading control. (e) Effect of miR-181a on intracellular CAMP content was detected in HT1080 cells transfected with the indicated plasmids. Each value represents the mean \pm S.D. of three independent experiments. ${ }^{* \star *} P<0.001$. (f) Effects of miR-181a on transactivation of RAR $\alpha$ were analyzed in COS7 cells co-transfected with RARE-TK-luciferase reporter and the indicated plasmids. Each value represents the mean \pm S.D. of three independent experiments. ${ }^{* *} P<0.01$

largely because of the fact that the majority of miRNAs can downregulate large numbers of target mRNAs. ${ }^{26}$ Thus, the involvement of miR-181 either as tumor suppressor or with oncogenic activities should depend on the specific genes it targeted in particular tissue or cell type. Here, we reported that miR-181a could target 3'UTR of AC9 and downregulate AC9 protein in APL cells, finally decreasing the intracellular CAMP level (Figure 7) and being a negative regulator for APL cell differentiation.

On the other hand, one gene could be also regulated by multiple miRNAs. ${ }^{27}$ Bioinformatics analysis predicted numerous potential miRNAs to be able to target AC9 mRNA. Here, we revealed the role of miR-181a in the downregulation of AC9 expression in APL cells. Nevertheless, we could not eliminate the possibility that other miRNAs, including $\mathrm{miR}-181 \mathrm{~b}$ and miR-181c, may also participate in AC9 expression regulation. Actually, it was reported that miR-142-3p could downregulate AC9 expression at the translational level and functionally control the production of cAMP in CD4 + CD25 - T cells and CD4 + CD25 + Treg cells. ${ }^{8}$ However, we did not find any significant difference in the expression of miR-142-3p in NB4 and NB4-R1 cells (data not shown). It should be therefore important to search for the combinations of miRNAs expressed in different cell types and focus on their coordinate regulation on cell-specific target genes. ${ }^{28,29}$

CEBP $\alpha$ is considered as a key factor for normal granulopoiesis. ${ }^{30}$ Here we found a repressive role of CEBP $\alpha$ on miR-181a expression. But we do not think that CEBP $\alpha$ is the only and the most important regulatory factor for miR-181a. This view is based on the following aspects: (1) several putative transcription factor-binding sites other 
a

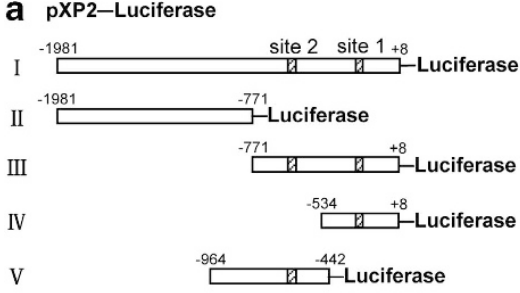

b
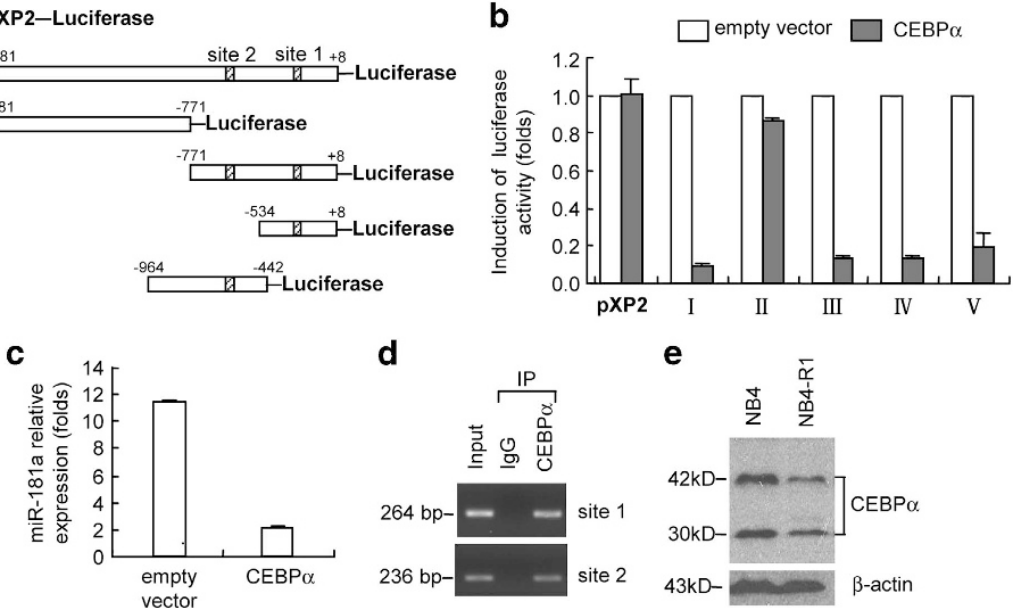

d

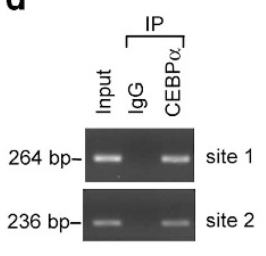

e

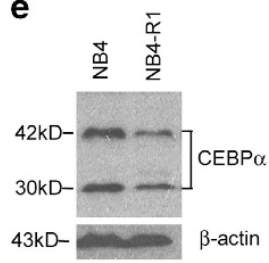

Figure 5 Negative regulation of CEBP $\alpha$ on miR-181a expression. (a) Schema of miR-181a promoter-containing $p X P 2-$ luciferase reporter constructs. Sites 1 and 2 are two potential CEBP $\alpha$-binding sites. (b) Effects of CEBP $\alpha$ on activation of different miR-181a promoter-containing reporter genes. Each value represents the mean \pm S.D. of three independent experiments. (c) miR-181a was co-transfected with CEBP $\alpha$ expression plasmid or empty vector into $293 \mathrm{~T}$ cells. miR-181a expression was analyzed by real-time quantitative PCR. Each value represents the mean \pm S.D. of three independent experiments. (d) ChIP assay was used to test the binding of CEBP $\alpha$ to miR-181a gene promoter in 293T cells transfected with CEBP $\alpha$ expression plasmid. (e) Western blot analysis of CEBP $\alpha$ expression in NB4 and NB4-R1 cells. $\beta$-Actin was used as a loading control
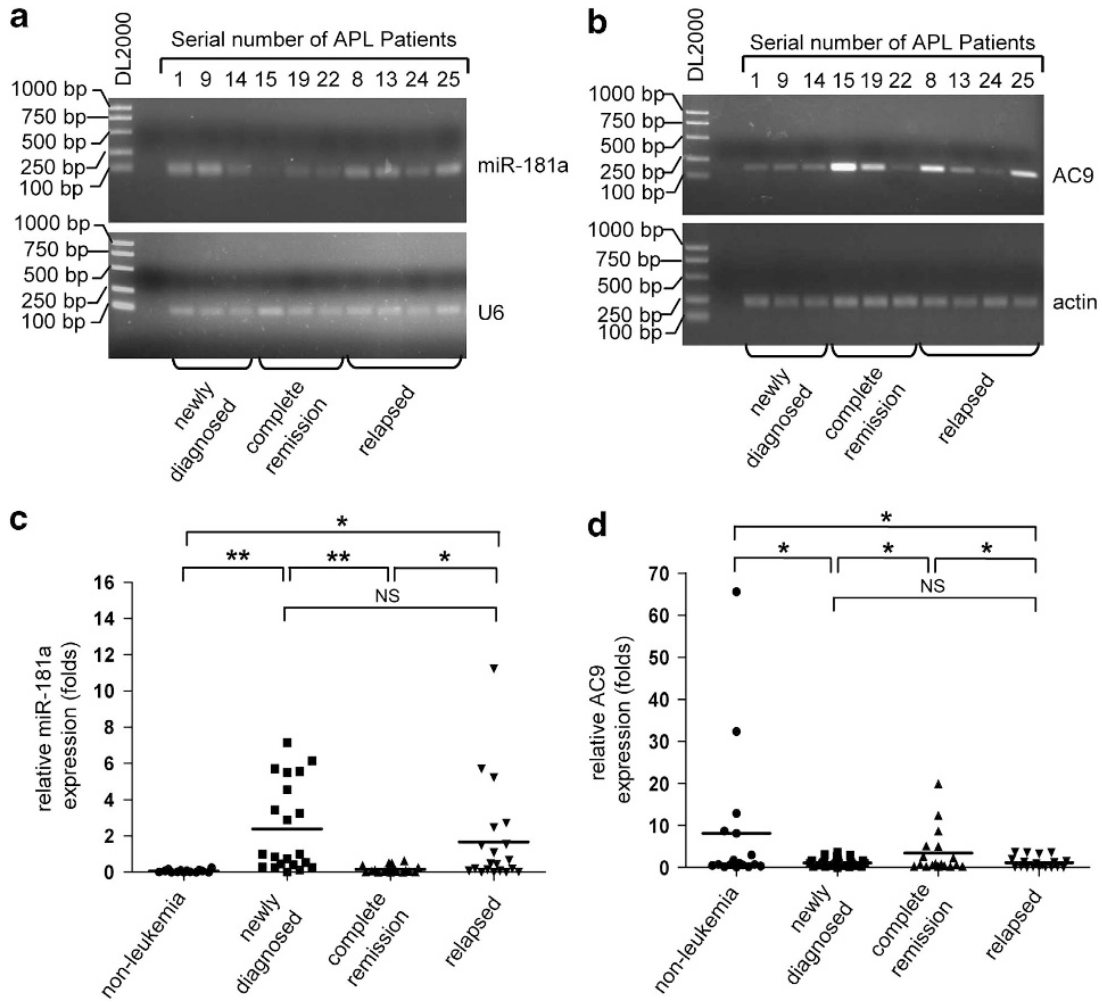

Figure 6 Expression of miR-181a and AC9 in fresh APL cells. (a and $\mathbf{b})$ RT-PCR analysis of miR-181a (a) and AC9 (b) expression in primary bone marrow cells from APL patients. (c and d) Real-time quantitative PCR analysis of miR-181a (c) and AC9 (d) expression in primary bone marrow cells from APL patients. Each PCR assay was performed in triplicate and three independent experiments were repeated. ${ }^{*} P<0.05$; ${ }^{*} P<0.01$; NS, not significant

than $\mathrm{CEBP} \alpha$ were found in the promoter region of miR-181a gene. (2) Quantitative RT-PCR analysis in bone marrow cells from patients showed no inverse correlation between $\mathrm{CEBP} \alpha$ and miR-181a expression (Figure $6 \mathrm{c}$ and Supplementary Figure S2). (3) Both $\operatorname{CEBP} \alpha$ and miR-181a levels were decreased after ATRA treatment in NB4 cells
(Supplementary Figure S3), ${ }^{31,32}$ not manifesting the inverse correlation either. Then, the molecular mechanisms underlying the expression regulation of miR-181a should be very complex. Our results that $\mathrm{CEBP} \alpha$ negatively regulated miR-181a expression may be associated with the differential expression of miR-181a and AC9 in NB4 and NB4-R1 cells. 


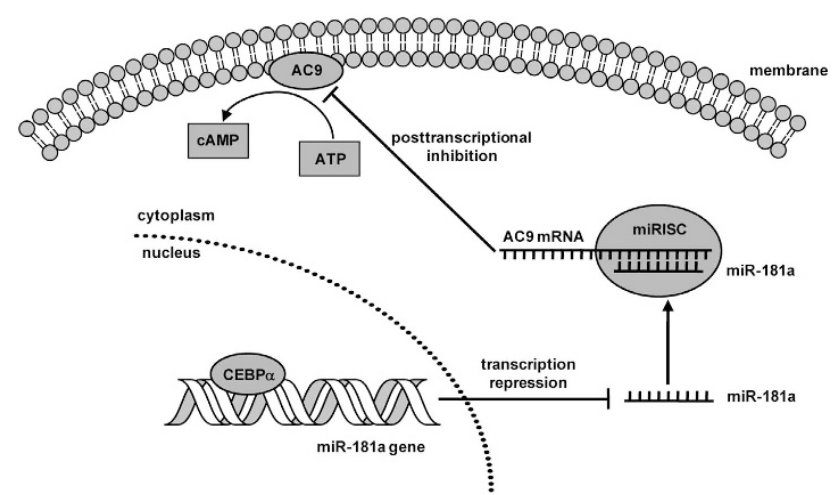

Figure 7 Summary diagram of expression regulation of AC9 gene. miR-181a could block AC9 expression at the post-transcriptional level through binding to the complementary sites in the $3^{\prime}$ UTR of AC9 mRNA, and decrease the intracellular CAMP content. The expression of miR-181a itself could be inhibited by CEBP $\alpha$

Finally, it was worth noting that, in addition to AC9 isoform, AC7 was also expressed in NB4 cells. According to the literature, AC7 is a ubiquitously expressed isoform. ${ }^{1}$ The distinct roles of $\mathrm{AC7}$ in hematopoiesis, brain and immune responses has been widely reported. ${ }^{33,34}$ We did not get more information in this study about the functions of AC7 in APL cell differentiation because of the inefficiency of antibodies.

Taken together, we reported here for the first time the implication of AC9 protein in ATRA-induced APL cell differentiation. We also revealed the downregulation of miR-181a on AC9 expression by targeting AC9 mRNA. Our study not only strongly supported the indispensable role of CAMP/PKA pathway in the therapeutic action of ATRA on APL, but also suggested the potential value of both AC9 and miR-181a in the clinical diagnosis and treatment of leukemia.

\section{Materials and Methods}

Cell culture. The cells NB4, NB4-R1, HT1080, COS7 and 293T were respectively cultured in RPMI 1640 or DMEM supplemented with $10 \%$ fetal bovine serum and $2 \mathrm{mmol} / \mathrm{l}$ L-glutamine. Fresh bone marrow cells were obtained with informed consent from APL patients. Leukemia cells were isolated and enriched on Ficoll solution. All cell cultures were incubated at $37^{\circ} \mathrm{C}$ in humidified air with $5 \% \mathrm{CO}_{2}$.

Plasmids. The expression plasmids AC9 and $\operatorname{CEBP} \alpha$ were constructed as follows: full-length CDNAs were amplified by RT-PCR and then subcloned into pcDNA3.1 vector. To generate AC9 $3^{\prime}$ UTR-containing reporter construct, a fragment of AC9 $3^{\prime}$ UTR including putative miR-181a complementary sequences was fused to a modified pcDNA3.1 vector, in which the luciferase gene had been inserted in the upstream of cloning sites. The mut-AC9 $3^{\prime}$ UTR-containing reporter plasmid was prepared by Mutagenesis Kit (Stratagene, La Jolla, CA, USA). The RARE-TK-Luciferase reporter plasmid was described previously. ${ }^{3}$ To prepare the miR-181a expression plasmid, a fragment including pre-miR-181a sequences was amplified from genomic DNA and subcloned into pcDNA3.1 vector. A series of miR-181a gene promoter-containing reporter plasmids were produced by inserting the different fragments from miR-181a2 gene promoter into pXP2-luciferease vector. ${ }^{35}$ All the constructs were verified by DNA sequencing.

Transfection and luciferase reporter assay. Transfection was performed by using SuperFect (Qiagen, Crawley, UK) or Profection Mammalian Transfection System Calcium Phosphate (Promega, Madison, WI, USA) according to the manufacturer's protocols. Luciferase activity was measured by DualLuciferase Assay System (Promega). pRL-TK plasmid (Promega) was used to normalize the transfection efficiency. Data were shown as the means \pm S.D. of three independent experiments.
RNA interference. To make siRNAs against human AC9 (si-AC9), two 21-bp sequences (5'-AAGGAGATGGTGAACATGAGA-3' and $5^{\prime}$-AACATACACTTCC CAGTGTTC- $3^{\prime}$ ) were selected and cloned into pSilencer3.1 vector, named as psi-AC9-(1) and psi-AC9-(2). psi-NC from Ambion (Austin, TX, USA) was used as a negative control. To establish AC9-knockdown stable cell lines, two si-AC9 were subcloned into a lentivirus vector pFU-GW-009 and then transfected into NB4 cells by lentivirus-based system. Positive clones were selected with $0.5 \mu \mathrm{g} / \mathrm{ml}$ puromycin (Sigma, St. Louis, MO, USA).

miRNA microarray. In all, $100 \mathrm{ng}$ of total RNA, prepared with mirVana miRNA extraction Kit (Ambion), was labeled and hybridized by using Human microRNA Microarray (Agilent Technologies, Santa Clara, CA, USA) following the manufacturer's protocol. Hybridization signals were detected with a DNA microarray scanner (Agilent Technologies) and the scanned images were analyzed using Agilent Feature Extraction software (v10.7). Raw data were normalized by Quantile algorithm, Gene Spring Software 11.0 (Agilent Technologies).

Reverse transcription-PCR and real-time quantitative PCR. Total RNA was extracted with Trizol (Invitrogen, Carlsbad, CA, USA). After reverse transcription, CDNA was amplified under the following conditions: $95^{\circ} \mathrm{C}$ for $5 \mathrm{~min}$; 23-33 cycles of $95^{\circ} \mathrm{C}$ for $30 \mathrm{~s}, 58^{\circ} \mathrm{C}$ for $30 \mathrm{~s}, 72^{\circ} \mathrm{C}$ for $30 \mathrm{~s}$; and $72^{\circ} \mathrm{C}$ for $10 \mathrm{~min}$. The primers used for RT-PCR analysis of AC isoforms (AC1-9) expression were listed in Supplementary Table S1. Other primers included: actin: $5^{\prime}$-CATCCT CACCCTGAAGTACCCC-3' and 5'-AGCCTGGATGCAACGTACATG-3'; AC9: $5^{\prime}$-AACAGCACCAAGGCTTCTGGAGGAC- $3^{\prime}$ and $5^{\prime}$-TCTTGAACCTCAGCGGAA GGAGAGC-3'; miR-181a: 5'-GCATCGAACATTCAACGCTGTCG-3' and 5'-TGCA GGGTCCGAGGTATT-3'; U6: 5'-TCGCTTCGGCAGCACATA-3' and 5'-TTTG CGTGTCATCCTTGC- $3^{\prime}$. For real-time quantitative PCR, CDNA was amplified by using SYBR-Green Premix (Takara, Otsu, Japan) under the conditions: $50^{\circ} \mathrm{C}$ for $2 \mathrm{~min} ; 95^{\circ} \mathrm{C}$ for $10 \mathrm{~min} ; 40$ cycles of $95^{\circ} \mathrm{C}$ for $15 \mathrm{~s}, 58^{\circ} \mathrm{C}$ for $30 \mathrm{~s}, 72{ }^{\circ} \mathrm{C}$ for $30 \mathrm{~s}$. AC9 and miR-181a levels were respectively normalized to the expression of actin and U6. The data were analyzed by deltaCt method.

Western blot analysis. Western blot was performed as described previously. ${ }^{35}$ The primary antibodies used in this study include: CEBP $\alpha$ and AC9 (Santa Cruz Biotechnology, Santa Cruz, CA, USA), Flag and $\beta$-actin (Sigma).

Determination of intracellular cAMP content. The intracellular cAMP content was determined by enzyme immunoassay (Assay Designs, Ann Arbor, MI, USA). Briefly, the cells were cultured without serum for $30 \mathrm{~min}$, and then treated with $0.5 \mathrm{mmol} / /$ phosphodiesterase inhibitor IBMX for $10 \mathrm{~min}$ at $37^{\circ} \mathrm{C}$. To terminate the reaction, $300 \mu \mathrm{l} 7.5 \%$ trichloroacetic acid was immediately added to cell pellets, and then $1 \mathrm{ml}$ ether was added. The upper ether was removed after vortex, and the CAMP content of cell lysates were detected following the manufacturer's instruction.

Chromatin immunoprecipitation. ChIP was performed using EZ ChIP kit (Millipore, Billerica, MA, USA) according to the instruction of the manufacturer. The primers specific to miR-181a2 gene promoter were as follows: site 1: $5^{\prime}$-GGCGTAGCAGATCCCCAATATATGTTA-3' and $5^{\prime}$-TGATGGATGCTCTTAC ATTCCTCTCTG-3'; site 2: 5'-CGACACCTCTGTGGTAACTTAGTAATG-3' and $5^{\prime}$-CTCACTCATCATACTGCTTCTGTTGTC-3'. Normal rabbit IgG (Santa Cruz Biotechnology) was used to control the nonspecific immunoprecipitation.

Statistical analysis. Student's t-test was used to evaluate the differences. For the data of patients from real-time quantitative PCR measurement, statistical analysis was performed by one-way ANOVA with the Newman-Keuls test.

\section{Conflict of Interest}

The authors declare no conflict of interest.

Acknowledgements. We thank all members of Shanghai Institute of Hematology for their support. This work was supported in part by National Natural Science Foundation of China (81170508); Leading Talent Program of Shanghai Municipal Human Resources and Social Security Bureau; and the Samuel Waxman Cancer Research Foundation. 
1. Sunahara RK, Taussig R. Isoforms of mammalian adenylyl cyclase: multiplicities of signaling. Mol Interv 2002; 2: 168-184.

2. Guillemin MC, Raffoux E, Vitoux D, Kogan S, Soilihi H, Lallemand-Breitenbach V et al. In vivo activation of cAMP signaling induces growth arrest and differentiation in acute promyelocytic leukemia. J Exp Med 2002; 196: 1373-1380.

3. Zhao Q, Tao J, Zhu Q, Jia PM, Dou AX, Li X et al. Rapid induction of CAMP/PKA pathway during retinoic acid-induced acute promyelocytic leukemia cell differentiation. Leukemia 2004; 18: 285-292.

4. Quenech'Du N, Ruchaud S, Khelef N, Guiso N, Lanotte M. A sustained increase in the endogenous level of cAMP reduces the retinoid concentration required for APL cell maturation to near physiological levels. Leukemia 1998; 12: 1829-1833.

5. Ruchaud S, Duprez E, Gendron MC, Houge G, Genieser HG, Jastorff B et al. Two distinctly regulated events, priming and triggering, during retinoid-induced maturation and resistance of NB4 promyelocytic leukemia cell line. Proc Natl Acad Sci USA 1994; 91 : 8428-8432.

6. Hacker BM, Tomlinson JE, Wayman GA, Sultana R, Chan G, Villacres E et al. Cloning, chromosomal mapping, and regulatory properties of the human type 9 adenylyl cyclase (ADCY9). Genomics 1998; 50: 97-104.

7. Cumbay MG, Watts VJ. Novel regulatory properties of human type 9 adenylate cyclase. J Pharmacol Exp Ther 2004; 310: 108-115.

8. Huang B, Zhao J, Lei Z, Shen S, Li D, Shen GX et al. miR-142-3p restricts cAMP production in CD4 + CD25- T cells and CD4 + CD25 + TREG cells by targeting AC9 mRNA. EMBO Rep 2009; 10: 180-185

9. Krol J, Loedige I, Filipowicz W. The widespread regulation of microRNA biogenesis, function and decay. Nat Rev Genet 2010; 11: 597-610.

10. Havelange V, Garzon R, Croce CM. MicroRNAs: new players in acute myeloid leukaemia. Br J Cancer 2009; 101: 743-748

11. Sun SM, Rockova V, Bullinger L, Dijkstra MK, Dohner H, Lowenberg B et al. The prognostic relevance of miR-212 expression with survival in cytogenetically and molecularly heterogeneous AML. Leukemia 2013; 27: 100-106.

12. Schotte $D$, Pieters $R$, Den Boer ML. MicroRNAs in acute leukemia: from biological players to clinical contributors. Leukemia 2012; 26: 1-12.

13. Shivdasani RA. MicroRNAs: regulators of gene expression and cell differentiation. Blood 2006; 108: 3646-3653

14. Calin GA, Dumitru CD, Shimizu M, Bichi R, Zupo S, Noch E et al. Frequent deletions and down-regulation of micro- RNA genes miR15 and miR16 at 13q14 in chronic lymphocytic leukemia. Proc Natl Acad Sci USA 2002; 99: 15524-15529.

15. Klein U, Lia M, Crespo M, Siegel R, Shen Q, Mo T et al. The DLEU2/miR-15a/16-1 cluster controls $\mathrm{B}$ cell proliferation and its deletion leads to chronic lymphocytic leukemia. Cancer Cell 2010; 17: 28-40.

16. Cimmino A, Calin GA, Fabbri M, lorio MV, Ferracin M, Shimizu M et al. miR-15 and miR-16 induce apoptosis by targeting BCL2. Proc Natl Acad Sci USA 2005; 102 13944-13949.

17. Duprez E, Ruchaud S, Houge G, Martin-Thouvenin V, Valensi F, Kastner P et al. A retinoid acid 'resistant' $t(15 ; 17)$ acute promyelocytic leukemia cell line: isolation, morphological, immunological, and molecular features. Leukemia 1992; 6: 1281-1287.

18. Chen CZ, Li L, Lodish HF, Bartel DP. MicroRNAs modulate hematopoietic lineage differentiation. Science 2004; 303: 83-86.

19. Wang X, Gocek E, Liu CG, Studzinski GP. MicroRNAs181 regulate the expression of p27Kip1 in human myeloid leukemia cells induced to differentiate by 1,25-dihydroxyvitamin D3. Cell Cycle 2009; 8: 736-741

20. Garzon R, Pichiorri F, Palumbo T, Visentini M, Ageilan R, Cimmino A et al. MicroRNA gene expression during retinoic acid-induced differentiation of human acute promyelocytic leukemia. Oncogene 2007; 26: 4148-4157.
21. Marcucci G, Radmacher MD, Maharry K, Mrozek K, Ruppert AS, Paschka P et al. MicroRNA expression in cytogenetically normal acute myeloid leukemia. $N$ Engl $J$ Med 2008; 358: 1919-1928.

22. Ji J, Yamashita T, Budhu A, Forgues M, Jia HL, Li C et al. Identification of microRNA-181 by genome-wide screening as a critical player in EpCAM-positive hepatic cancer stem cells. Hepatology 2009; 50: 472-480.

23. Ke G, Liang L, Yang JM, Huang X, Han D, Huang S et al. MiR-181a confers resistance of cervical cancer to radiation therapy through targeting the pro-apoptotic PRKCD gene. Oncogene 2013; 32: 3019-3027.

24. Shi L, Cheng Z, Zhang J, Li R, Zhao P, Fu Z et al. hsa-mir-181a and hsa-mir-181b function as tumor suppressors in human glioma cells. Brain Res 2008; 1236: 185-193.

25. Zhu W, Shan X, Wang T, Shu Y, Liu P. miR-181b modulates multidrug resistance by targeting BCL2 in human cancer cell lines. Int J Cancer 2010; 127: 2520-2529.

26. Lim LP, Lau NC, Garrett-Engele P, Grimson A, Schelter JM, Castle J et al. Microarray analysis shows that some microRNAs downregulate large numbers of target mRNAs. Nature 2007; 433: 769-773.

27. Krek A, Grun D, Poy MN, Wolf R, Rosenberg L, Epstein EJ et al. Combinatorial microRNA target predictions. Nat Genet 2005; 37: 495-500.

28. Su SF, Chang YW, Andreu-Vieyra C, Fang JY, Yang Z, Han B et al. miR-30d, miR-181a and miR-199a-5p cooperatively suppress the endoplasmic reticulum chaperone and signaling regulator GRP78 in cancer. Oncogene 2013; 32: 4694-4701.

29. Zhang S, Li Q, Liu J, Zhou XJ. A novel computational framework for simultaneous integration of multiple types of genomic data to identify microRNA-gene regulatory modules. Bioinformatics 2011; 27: i401-i409.

30. Mueller BU, Pabst T. C/EBPalpha and the pathophysiology of acute myeloid leukemia. Curr Opin Hematol 2006; 13: 7-14.

31. Liu TX, Zhang JW, Tao J, Zhang RB, Zhang QH, Zhao CJ et al. Gene expression networks underlying retinoic acid-induced differentiation of acute promyelocytic leukemia cells. Blood 2000; 96: 1496-1504.

32. Saumet A, Vetter G, Bouttier M, Portales-Casamar E, Wasserman WW, Maurin T et al. Transcriptional repression of microRNA genes by PML-RARA increases expression of key cancer proteins in acute promyelocytic leukemia. Blood 2009; 113: 412-421.

33. Duan B, Davis R, Sadat EL, Collins J, Sternweis PC, Yuan D et al. Distinct roles of adenylyl cyclase VII in regulating the immune responses in mice. J Immunol 2010; 185: 335-344.

34. Joeyen-Waldorf J, Nikolova YS, Edgar N, Walsh C, Kota R, Lewis DA et al. Adenylate cyclase 7 is implicated in the biology of depression and modulation of affective neural circuitry. Biol Psychiatry 2012; 71: 627-632.

35. Xiao S, Li D, Zhu HQ, Song MG, Pan XR, Jia PM et al. RIG-G as a key mediator of the antiproliferative activity of interferon-related pathways through enhancing p21 and p27 proteins. Proc Natl Acad Sci USA 2006; 103: 16448-16453.

(i) (-) $\Theta$ Cell Death and Disease is an open-access journal By ${ }^{\mathrm{NC}}$ no published by Nature Publishing Group. This work is licensed under a Creative Commons Attribution-NonCommercialNoDerivs 3.0 Unported License. The images or other third party material in this article are included in the article's Creative Commons license, unless indicated otherwise in the credit line; if the material is not included under the Creative Commons license, users will need to obtain permission from the license holder to reproduce the material. To view a copy of this license, visit http://creativecommons.org/licenses/ by-nc-nd/3.0/

Supplementary Information accompanies this paper on Cell Death and Disease website (http://www.nature.com/cddis) 